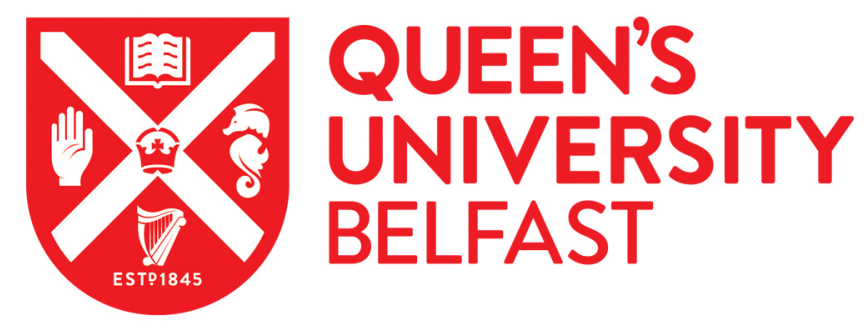

\title{
Ion acceleration and plasma jet formation in ultra-thin foils undergoing expansion and relativistic transparency
}

King, M., Gray, R. J., Powell, H. W., MacLellana, D. A., Gonzalez-Izquierdo, B., Stockhausen, L. C., Hicks, G. S., Dover, N. P., Rusby, D. R., Carroll, D. C., Padda, H., Torres, R., Kar, S., Clarke, R. J., Musgrave, I. O., Najmudin, Z., Borghesi, M., Neely, D., \& McKenna, P. (2016). Ion acceleration and plasma jet formation in ultrathin foils undergoing expansion and relativistic transparency. Nuclear Instruments \& Methods in Physics Research - Section A: Accelerators, Spectrometers, Detectors, and Associated Equipment, 829, $163-166$. https://doi.org/10.1016/j.nima.2016.02.032

Published in:

Nuclear Instruments \& Methods in Physics Research - Section A: Accelerators, Spectrometers, Detectors, and Associated Equipment

\section{Document Version:}

Early version, also known as pre-print

Queen's University Belfast - Research Portal:

Link to publication record in Queen's University Belfast Research Portal

\section{Publisher rights}

Copyright 2016 The Authors

\section{General rights}

Copyright for the publications made accessible via the Queen's University Belfast Research Portal is retained by the author(s) and / or other copyright owners and it is a condition of accessing these publications that users recognise and abide by the legal requirements associated with these rights.

Take down policy

The Research Portal is Queen's institutional repository that provides access to Queen's research output. Every effort has been made to ensure that content in the Research Portal does not infringe any person's rights, or applicable UK laws. If you discover content in the Research Portal that you believe breaches copyright or violates any law, please contact openaccess@qub.ac.uk. 


\title{
Ion acceleration and plasma jet formation in ultra-thin foils undergoing expansion and relativistic transparency
}

\author{
M. King ${ }^{\mathrm{a}}$, R. J. Gray ${ }^{\mathrm{a}}$, H. W. Powell ${ }^{\mathrm{a}}$, D. A. MacLellan ${ }^{\mathrm{a}}$, B. Gonzalez-Izquierdo ${ }^{\mathrm{a}}$, L. C. Stockhausen ${ }^{\mathrm{b}}$, G. Hicks $^{\mathrm{c}}$, N. \\ P. Dover ${ }^{\mathrm{c}}$, D. R. Rusby ${ }^{\mathrm{a}, \mathrm{d}}$, D. C. Carroll ${ }^{\mathrm{d}}$, H. Padda ${ }^{\mathrm{a}}$, R. Torres ${ }^{\mathrm{b}}$, S. Kare, R. J. Clarke ${ }^{\mathrm{d}}$, I. O. Musgrave ${ }^{\mathrm{d}}$, Z. \\ Najmudin $^{\mathrm{c}}$, M. Borghesi ${ }^{\mathrm{e}}$, D. Neely ${ }^{\mathrm{d}}$, P. McKenna ${ }^{\mathrm{a}}$ \\ ${ }^{a}$ SUPA Department of Physics, University of Strathclyde, Glasgow G4 ONG, UK \\ ${ }^{b}$ Centro de Laseres Pulsados (CLPU), Parque Cientifico, Calle del Adaja s/n. 37185 Villamayor, Salamanca, Spain \\ ${ }^{c}$ The John Adams Institute for Accelerator Science, Blackett Laboratory, Imperial College London, London SW7 2BZ, UK \\ ${ }^{d}$ Central Laser Facility, STFC Rutherford Appleton Laboratory, Oxfordshire OX11 OQX, UK \\ ${ }^{e}$ Centre for Plasma Physics, Queens University Belfast, Belfast BT7 1NN, UK
}

\begin{abstract}
At sufficiently high laser intensities, rapid heating and decompression of electrons in an ultra-thin target foil can result in the target becoming relativistically transparent to the laser light during the interaction. Ion-acceleration in this regime is strongly affected by the transition from an opaque to a relativistically transparent plasma. By spatially resolving the laser-accelerated proton beam at near-normal laser incidence and at an incidence angle of $30^{\circ}$, we identify characteristic features both experimentally and in particle-in-cell simulations which are consistent with the onset of three distinct ion acceleration mechanisms: sheath acceleration; radiation pressure acceleration; and transparencyenhanced acceleration. The latter mechanism occurs late in the interaction and is mediated the formation of a plasma jet extending into the expanding ion population. The effect of laser incident angle on the plasma jet is explored.
\end{abstract}

\section{Introduction}

Intense laser-driven ion acceleration from thin foils offers a route towards the creation of compact, high energy, short pulse ion sources. These sources could potentially be applied to ion oncology and the fast ignition approach to inertial confinement fusion $[1,2]$. Over the past 15 years, the target normal sheath acceleration (TNSA) mechanism [3] has been investigated as a primary acceleration mechanism and whilst much progress has been made, the spectral control and high maximum energies required (particularly for oncology) has not yet been achieved $[4,5]$. Recent advances in ultra-thin foil targetry and enhancements in laser peak intensity and contrast have led to investigations of new acceleration mechanisms, with promising potential for ion energy scaling and spectral and divergence control.

The irradiation of sub- $\mu$ m-thick foils with ultraintense $\left(>10^{20} \mathrm{Wcm}^{-2}\right)$ laser pulses can result in a variety of ion acceleration mechanisms. The TNSA mechanism will typically occur early on the rising

Email address: paul.mckenna@strath.ac.uk (P. McKenna) edge of the laser pulse as target electrons at the target front side are heated and drive the formation of sheath fields. As the laser intensity continues to increase on the rising edge, the radiation pressure acceleration (RPA) mechanism [6], in which the target surface is directly driven forward due to the pressure of the incident laser radiation, can occur. This mechanism is predicted to produce an ion beam with a narrow energy spectrum, low divergence and a favourable energy scaling $[7,8]$. In the case of ultra-thin (nanometer-scale) foil targets, the RPA mechanism can become unstable to Rayleigh-Taylor-like transverse instabilities, resulting in bubble-like structures in the resulting proton beam [9]. If during the interaction the plasma electron population decompresses to the extent that the target undergoes relativistic-induced transparency (RIT) [10], RPA ceases and the remainder of the laser pulse propagates through the target. This gives rise to volumetric heating of the target electrons, which can enhance the energy of the TNSA accelerated ions. This mechanism is referred to as transparency-enhanced acceleration or the breakout afterburner (BOA) scheme [11,12]. The collective plasma electron response to the onset of RIT can give rise to asymmetric electron beam distributions [13] and 


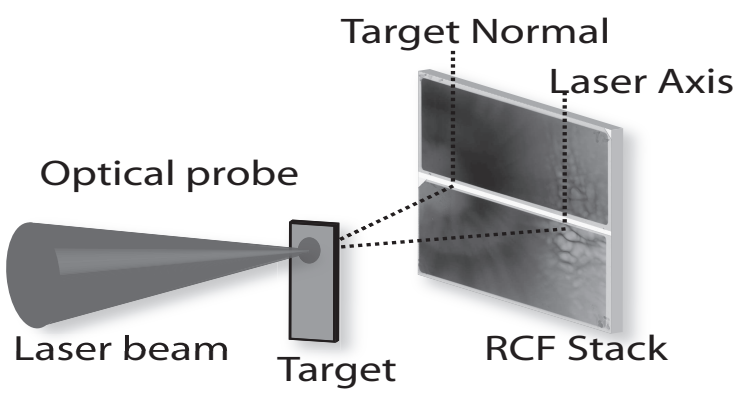

Figure 1: Schematic illustrating the experimental arrangement.

controllable plasma structures [14]. We have recently shown that in the case of relatively long (hundreds of femtoseconds) laser pulses, the plasma can expand tens of microns during the laser pulse interaction, giving rise to conditions in which a jet of high energy electrons can be produced, driving enhanced laser energy coupling in a localised region of the sheath-acceleration ion population [15].

In this article, following on from results reported in our earlier paper [15], we report on the influence of the angle of incidence of the laser light on the formation of the plasma jet and the complex dynamics occurring during the onset of RIT.

\section{Experiment results}

Using the Vulcan petawatt laser at the Rutherford Appleton Laboratory, pulses of $1.054 \mu \mathrm{m}$-wavelength light, with (1.0 \pm 0.2$)$ ps duration (full width at half maximum $(\mathrm{FWHM})$ ) and $(200 \pm 15) \mathrm{J}$ energy were focused onto an aluminium target with a thickness of $10 \mathrm{~nm}$. The spot size was $7.3 \mu \mathrm{m}$ (FWHM) producing a peak intensity of $2 \times 10^{20} \mathrm{Wcm}^{-2}$. A plasma mirror was employed to improve the laser intensity contrast by a factor of $\approx 100$. The angle of incidence of the laser light with respect to the target normal was set at either near-normal $\left(0^{\circ}\right)$ or $30^{\circ}$. In all cases the laser pulse was p-polarized.

The focus of the experimental results presented here is the proton spatial-intensity profile, as measured using a stack of radiochromic (dosimetry) film (RCF). The stack contains filters which enables the energy of the protons stopped at each RCF layer to be set. It was positioned $7.5 \mathrm{~cm}$ behind the target with the centre off-set to position the laser axis close to one side as illustrated in Fig. 1. Both the beams of protons accelerated along the laser axis and target normal can be detected on the same RCF stack when the incident angle is changed to $30^{\circ}$. A narrow slot along the central horizontal axis of

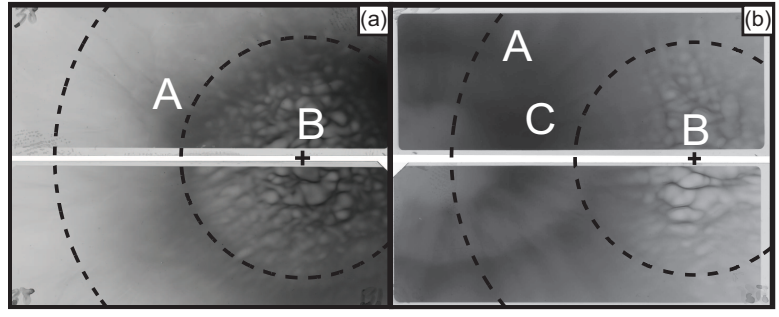

Figure 2: Proton spatial-intensity profiles in the energy range 5-7 $\mathrm{MeV}$, for a $10 \mathrm{~nm}$-thick aluminium target at an laser incident angle to the target of: (a) $0^{\circ}$ (b) $30^{\circ}$. Dashed circular lines indicate radii at $15^{\circ}$ and $30^{\circ}$ centred on the laser propagation axis. Feature $\mathrm{A}$ indicates a ring-like distribution around target normal, $\mathrm{B}$ indicates bubble-like structures and feature $\mathrm{C}$ indicates a localised, higher proton energy feature.

the stack enabled ion energy spectra measurements using a Thomson parabola spectrometer.

Example measurements of the proton spatialintensity distribution, obtained with a $10 \mathrm{~nm}$-thick aluminium target irradiated at $0^{\circ}$ and $30^{\circ}$ incident angle is shown in Fig 2. The dashed lines are reference circles corresponding to $15^{\circ}$ and $30^{\circ}$ with respect to the laser propagation axis. From these measurements, it is clearly observed that different proton beam features, produced by the different acceleration mechanisms, are separated when the target is irradiated at an oblique angle. Three features are observed, and labelled A, B and $\mathrm{C}$ in Fig. 2 to aid the discussion below.

The annular ring like distribution (labeled Feature A) is consistent with proton spatial profiles previously measured in targets undergoing RIT $[12,16]$. For this particular target, the ring has a divergence half-angle of $\sim 12^{\circ}$. From 2(a), feature A is centred directly along the laser propagation axis for an incidence angle of $0^{\circ}$. The protons present in this feature have been driven by TNSA at the rear of the target. This is demonstrated in Fig. 2(b) when the angle of incidence is changed to $30^{\circ}$. In this case, the annular ring-like structure is still present with a similar divergence half-angle, centred at $\sim 30^{\circ}$ which is along the target normal axis.

Feature B comprises small bubble-like structures, similar to that observed due to the transverse instabilities associated with RPA [9]. In Fig 2(a) these bubblelike structures are contained within a circular area up to $\sim 15^{\circ}$ around the laser propagation axis. As these are structures associated with RPA in an expanding plasma, they are observed along the laser axis in Fig 2(b). The bubbles appear slightly elongated along the laser polarization axis, suggesting an additional effect from interacting at a non-normal incidence.

Feature $\mathrm{C}$ is difficult to distinguish at near-normal in- 


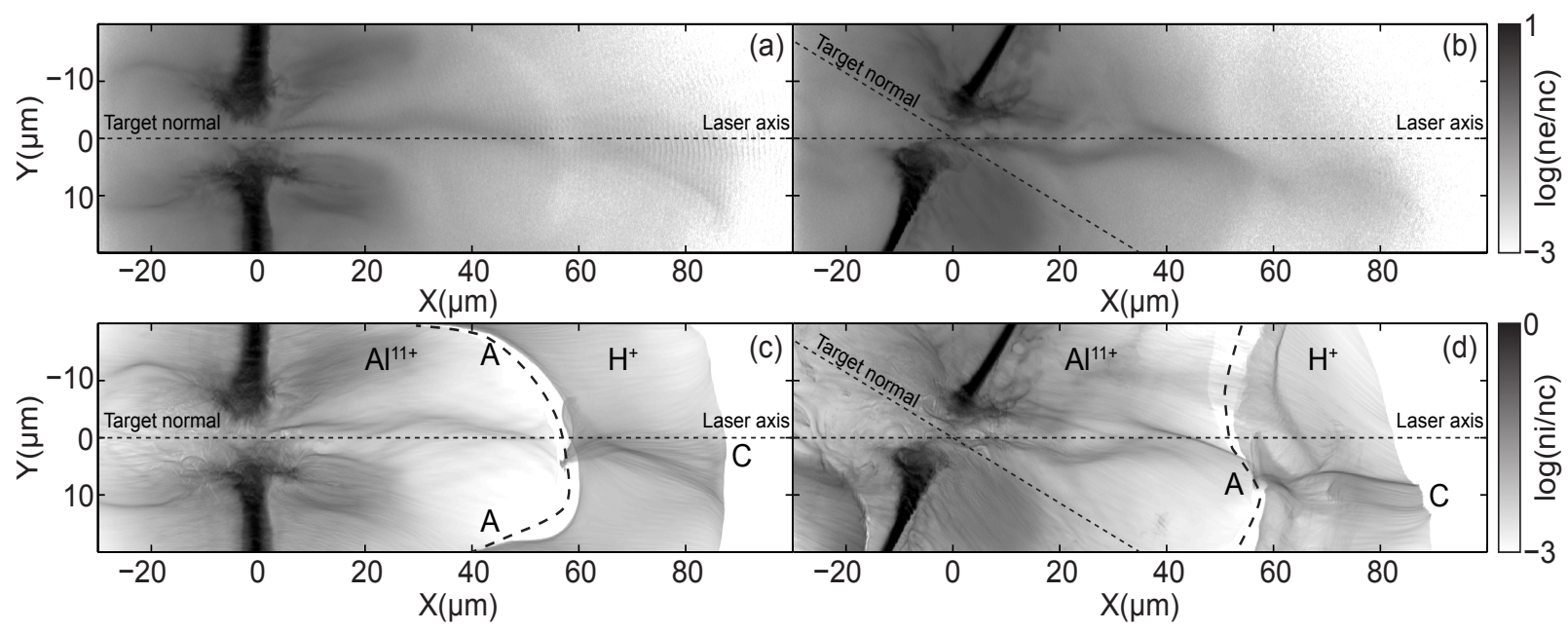

Figure 3: Top row: 2D PIC results showing electron density for the target initialised at (a) $0^{\circ}$ and (b) $30^{\circ}$ incident angle to the laser. Bottom row: density of the $\mathrm{Al}^{11+}$ and $\mathrm{H}^{+}$ions initialised at (c) $0^{\circ}$ and (d) $30^{\circ}$ incident angle to the laser. All plots are shown at an example time of 0.3 ps after the peak of the laser pulse has reached the target surface. The laser pulse is incident from the left along the $\mathrm{Y}=0$ axis. The dotted lines mark the laser and target normal axes.

cidence in Fig. 2(a) as it overlaps with the bubble-like structures. When irradiating at $30^{\circ}$ incidence angle, a strong feature can be seen between the target normal and the laser axes. This is associated with the formation of an electron jet from the rear of the target that is created in the expanding plasma as the target undergoes RIT [15]. This jet feature is found to be susceptible to hosing and is observed to vary in position from shot to shot. It is thus problematic to measure the ion energy spectrum produced by this feature using a fixed spectrometer sampling a small solid angle.

\section{Simulation results}

To investigate the features observed experimentally, 2D and 3D PIC simulations were undertaken using the EPOCH code [17]. For the 2D simulations, the simulation box was defined as $130 \mu \mathrm{m} \times 72 \mu \mathrm{m}$, with 26000 $\times 7200$ mesh cells. The simulations were run at $40 \mathrm{~nm}$ due to computational constraints in resolving the skin depth effects. The main target was initialised with an electron density $\mathrm{n}_{e}=630 \mathrm{n}_{c}$ (where $\mathrm{n}_{c}$ is the critical density) neutralised with the $\mathrm{Al}^{11+}$ ions. A neutral layer of $12 \mathrm{~nm} \mathrm{H}^{+}$ions with $\mathrm{n}_{e}=60 \mathrm{n}_{c}$ is initialised on the rear of the target, to produce the source of protons. The initial electron temperature for both the target and the surface layer is set to $10 \mathrm{keV}$. To simplify the simulation, there is no front surface layer and no carbon or oxygen species present. Simulations are undertaken with the target at both normal incidence to the laser and at a $30^{\circ}$ incidence. The incoming laser pulse was linearly polarised along the Y-axis and focused to Gaussian profile with a FWHM of $5 \mu \mathrm{m}$ at the front of the target (defined as $\mathrm{X}=0 \mu \mathrm{m}$ ) with the temporal profile defined as a Gaussian pulse with a FWHM of 570 fs.

In the $3 \mathrm{D}$ simulations, the simulation box was defined as $55 \mu \mathrm{m} \times 14.4 \mu \mathrm{m} \times 14.4$ with $2000 \times 360 \times$ 360 mesh cells. Due to the reduction of mesh resolution the target $\left(\mathrm{Al}^{11+}\right)$ and contamination layer $\left(\mathrm{H}^{+}\right)$was pre-expanded to a Gaussian spatial profile along the target normal axis with a peak electron density of $\mathrm{n}_{e}=53 \mathrm{n}_{c}$ and $\mathrm{n}_{e}=5 \mathrm{n}_{c}$ maintaining the same areal density as the $2 \mathrm{D}$ simulation. Simulations were run with the target at both $0^{\circ}$ and $30^{\circ}$ incidence. The laser pulse temporal duration was slightly reduced to $500 \mathrm{fs}$ and the spatial profile was focused to a 2D Gaussian profile with a FWHM of $5 \mu \mathrm{m}$ at the front of the target, again linearly polarised along the Y-axis.

Fig. 3 shows example density plots of the electrons (a)-(b) and ions (c)-(d) for simulations at both $0^{\circ}$ and $30^{\circ}$ incidence angle, $0.3 \mathrm{ps}$ after the peak of the laser pulse has interacted with the target. In both cases the laser pulse enters the system at $X=-30 \mu \mathrm{m}$, with the spatial profile centred at $\mathrm{Y}=0 \mu \mathrm{m}$. As the laser pulse interacts with the target, expansion of both the main $\mathrm{Al}^{11+}$ target and $\mathrm{H}^{+}$contaminant layer occurs. Due to their higher charge-to-mass ratio, the $\mathrm{H}^{+}$ions expand in front of the $\mathrm{Al}^{11+}$ ions. The highest energy $\mathrm{Al}^{11+}$ ions push against the rear of the $\mathrm{H}^{+}$layer, driving them out radially. This forms the ring-like feature A, as observed in 


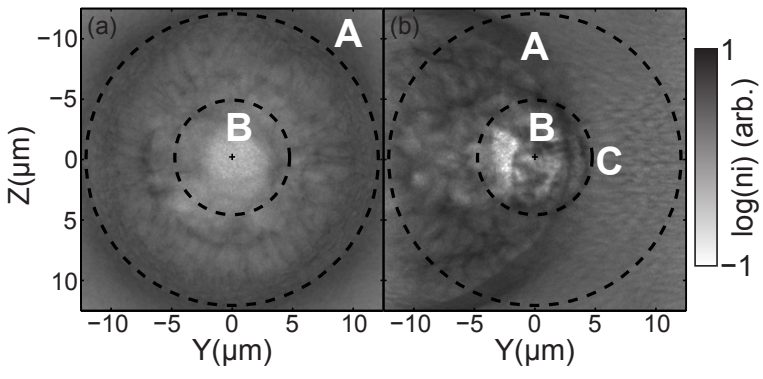

Figure 4: 3D results showing a summation of the proton density in the $\mathrm{Y}-\mathrm{Z}$ plane for energies $<15 \mathrm{MeV}$ with the target initialised at (a) $0^{\circ}$ and (b) $30^{\circ}$ incidence.

the RCF data. Further investigation of this effect will be the subject of future work. As this process is formed due to the target normal expansion of the ions, feature A can always been seen to be centred around the target normal direction for both the $0^{\circ}$ and $30^{\circ}$ incidence angle cases.

A plasma jet structure, corresponding to feature $\mathrm{C}$, is clearly observed at both incident angles. As discussed in Powell et al. [15], this jet is formed as the target undergoes RIT, and is contained by a self-generated azimuthal magnetic field. The portion of the laser pulse which is transmitted through the remainder of the target during RIT directly accelerates electrons in the jet to energies higher than the background electrons heated earlier in the laser pulse interaction. This in turn results in a localised energy increase in the sheath-accelerated ion population [15]. When the laser is at near-normal incidence, the jet is primarily directed along the common laser and target normal axis over a distance of $\sim 50 \mu \mathrm{m}$, into the $\mathrm{H}^{+}$layer, before it becomes subject to a hosinglike instability as the plasma density decreases. For the case of $30^{\circ}$ incidence, the jet is initially directed along the laser axis, but deviates more quickly due to the local plasma density asymmetry around it. The enhancement in the proton energy thereby typically occurs at an angle between the laser axis and the target normal direction. We note that in the experiment results, feature $\mathrm{C}$ is always observed at angle between these two axes when irradiating the target at $30^{\circ}$ incidence.

The overall results for the 3D simulations are in good agreement with the 2D simulations [15] and enable the proton distribution in the $\mathrm{Y}-\mathrm{Z}$ plane (the plane of the detector) to be plotted, enabling feature $\mathrm{B}$ to be more clearly observed, as seen in Fig 4. This figure shows the density of the summation of the lower energy band $(<15$ $\mathrm{MeV}$ ) of the protons and thus enables a comparison with the RCF measurements in Fig 2. Fig 4(a) shows the $0^{\circ}$ incidence case, and looks very similar to that of Fig 2(a).
The ring-like feature $\mathrm{A}$ is again centred along the laser axis and contains an unstable structure similar to that of feature B. At this incidence angle it is again difficult to observe feature $\mathrm{C}$ as it overlaps with the bubble-like instability structure. At $30^{\circ}$ incidence angle, these features can be resolved in Fig 4(b). The ring-like structure has clearly moved to be centred along the target normal direction. In the experimental case, the ions have $7.5 \mathrm{~cm}$ distance to travel before reaching the RCF diagnostic, whereas in the $3 \mathrm{D}$ simulations they are diagnosed at an approximate distance of $20 \mu \mathrm{m}$ from the target. This results in the ring-like structure overlapping with the laser axis features. The unstable structures remain centred along the laser axis, although the overlapping distributions make this difficult to clearly resolve. The short sample distance from the target means that the jet (feature C) also overlaps with the edge of feature A (a longer propagation distance is required for the jet to steer offaxis). Nevertheless, the localised proton energy is enhanced in this region due to the presence of the electron jet, as discussed in reference [15].

\section{Summary}

In summary, the interaction of an ultraintense laser pulse with an ultrathin foil target undergoing expansion and RIT has been investigated experimentally and numerically. When irradiating the target at an oblique angle of incidence, three distinct components are observed in the spatial-intensity profile of proton beam, which have characteristic signatures of three distinct ion acceleration mechanisms. The effect of the laser incidence angle on the characteristics of a plasma jet generated during the onset of RIT has been explored. The electrons in this jet are directly accelerated to higher energy by the laser pulse and couple additional energy to a local region of the sheath-accelerated proton distribution. The effect of the laser incidence angle on the jet formation, direction and energy coupling to ions will be investigated in more detail in a future experiment.

\section{References}

[1] H. Daido, et al., Rep. Prog. Phys. 75 (2012) 056401, and references therein.

[2] A. Macchi, et al., Rev. Mod. Phys. 85 (2013) 751, and references therein.

[3] S. Wilks, et al., Phys. Plasmas 8 (2001) 542

[4] J. Fuchs, et al., Nat. Phys. 2 (2006) 48-54.

[5] L. Robson, et al., Nat. Phys. 3 (2007) 58-62.

[6] T. Esirkepov, et al., Phys. Plasmas 92 (2004) 175003.

[7] A. Macchi, et al., Phys. Plasmas 92 (2004) 175003.

[8] S. Kar, et al., Phys. Rev. Lett. 109 (2012) 185006.

[9] C. Palmer, et al., Phys. Rev. Lett. 108 (2012) 225002. 
[10] V. Vshivkov, et al., Phys. Plasmas 5 (1998) 2727.

[11] L. Yin, et al., Laser Part. Beams 24 (2006) 291.

[12] D. Jung, et al., New. J. Phys. 15 (2013) 123035.

[13] R. Gray, et al., New. J. Phys. 16 (2014) 093027.

[14] B. Gonzalez-Izquierdo, et al., Nat. Phys. at press.

[15] H. Powell, et al., New. J. Phys. 17 (2015) 103033.

[16] N. Dover, et al., http://arxiv.org/abs/1406.3540.

[17] T. Arber, et al., Plasma Phys. Control. Fusion 57 (2015) 113001. 